\title{
STACS: New Active Contour Scheme for Cardiac MR Image Segmentation
}

\author{
Charnchai Pluempitiwiriyawej*, José M. F. Moura, Fellow, IEEE, Yi-Jen Lin Wu, and Chien Ho
}

\begin{abstract}
The paper presents a novel stochastic active contour scheme (STACS) for automatic image segmentation designed to overcome some of the unique challenges in cardiac MR images such as problems with low contrast, papillary muscles, and turbulent blood flow. STACS minimizes an energy functional that combines stochastic region-based and edge-based information with shape priors of the heart and local properties of the contour. The minimization algorithm solves, by the level set method, the Euler-Lagrange equation that describes the contour evolution. STACS includes an annealing schedule that balances dynamically the weight of the different terms in the energy functional. Three particularly attractive features of STACS are: 1) ability to segment images with low texture contrast by modeling stochastically the image textures; 2) robustness to initial contour and noise because of the utilization of both edge and region-based information; 3) ability to segment the heart from the chest wall and the undesired papillary muscles due to inclusion of heart shape priors. Application of STACS to a set of 48 real cardiac MR images shows that it can successfully segment the heart from its surroundings such as the chest wall and the heart structures (the left and right ventricles and the epicardium.) We compare STACS' automatically generated contours with manually-traced contours, or the "gold standard," using both area and edge similarity measures. This assessment demonstrates very good and consistent segmentation performance of STACS.
\end{abstract}

Index Terms-Active contour, cardiac magnetic resonance imaging (cardiac MRI), chamfer method, energy minimization, image segmentation, level set, shape and area similarities, stochastic model, stochastic relaxation.

\section{INTRODUCTION}

$\mathbf{M}$ AGNETIC resonance imaging (MRI) is a noninvasive tool that can be used to measure the deformation of an in vivo heart [1]-[3] and to help diagnose the presence of heart disease by analyzing the heart function throughout the cardiac cycle. To make this possible, it is necessary to segment various parts of the heart chambers in a magnetic resonance (MR) image sequence. In clinical studies, the segmentation task, particularly

Manuscript received June 1, 2004; revised December 27, 2004. This work was supported by the National Institutes of Health (NIH) under Grant R01EB/AI00318 and Grant P41EB001977 to the Pittsburgh NMR Center for Biomedical Research, CMU. The Associate Editor responsible for coordinating the review of this paper and recommending its publication was A. Amini. Asterisk indcates corresponding author.

${ }^{*}$ C. Pluempitiwiriyawej is with the Department of Electrical Engineering, Chulalongkorn University, Pratumwan, Bangkok, Thailand 10330 (e-mail: Charnchai.P@eng.chula.ac.th).

J. M. F. Moura is with the Department of Electrical and Computer Engineering, Carnegie Mellon University, Pittsburgh, PA 15213 USA (e-mail: moura@ece.cmu.edu).

Y.-J. L. Wu and C. Ho are with the Pittsburgh NMR Center for Biomedical Research, Carnegie Mellon University, Mellon Institute, Pittsburgh, PA 15213 USA.

Digital Object Identifier 10.1109/TMI.2005.843740 delineating the epicardium and the left and right ventricular endocardia, is often performed manually, which is tedious and time consuming. Thus, in practice, the analysis of the MR data is limited to a short time sequence per cardiac cycle. To help expedite this process and facilitate the analysis of more comprehensive MR data sets, an automatic and more quantitative approach for analyzing cardiac MR sequence data is highly desirable. However, to delineate the structures of interest and discriminate them from the background automatically poses considerable challenges.

Most active contour methods [4]-[7] for medical image segmentation are sensitive to noise and initialization due to their purely edge-based nature. The initial contour must start close enough to the true boundary for the contour to evolve. Moreover, turbulent blood flow in some MR images often causes faulty edges. The papillary muscles and other anatomy parts like the chest wall pose additional challenges for segmentation because, although they should be excluded, their MR image texture is very similar to the myocardium's texture. To overcome these problems, we find that it is important to include any available prior shape knowledge about the structures to be segmented.

This paper describes a novel stochastic active contour scheme (STACS) for cardiac image segmentation. This scheme is designed especially to address some of the unique problems in cardiac MR images. We opt for an energy minimization approach [8] that combines characteristics of curve evolution theory and statistical modeling of the images to segment a homogeneous object (the heart and its structures) from the background (chest wall or other anatomy). STACS has three particularly attractive features. First, it models the images as samples from random fields. This stochastic model can be applied to a large range of images, in particular, when contrast between distinct regions is difficult to distinguish by the human eye, and other methods may fail [7], [9]. Second, our method is region-based as well as edge-based. While the evolving contour attracts to strong edge points due to the presence of edge-information, the region-based information helps propagating the contour where edge information is missing. As a result, our method is less sensitive to the contour's initial condition than many previous purely edge-based snake algorithms. Third, we incorporate prior knowledge about the shape of the object (the heart and its structures) into the segmentation scheme, which helps to segment the heart from the chest wall or the myocardium from the papillary muscles. From our experience, to achieve successful segmentation of the heart and its chambers, the energy functional should adapt dynamically so that, for example, contrast and texture gradients can be the main drivers in the initial steps of the segmentation, while (loose) shape priors can become more significant toward the end. We achieve this by automatically relaxing the 
relative weight of the different terms in the energy functional through an annealing schedule. Several shape-based segmentation methods such as [10]-[12] have recently been described in the literature. Most of these methods are statistical-based and need a good training set. However, our approach to include shape priors is parametric and adjusts the shape by estimating the shape parameters. For example, the object shapes are assumed to be approximated by ellipses, which are easily parameterized and no training sets needed.

Finally, we illustrate the performance of STACS by segmenting six cardiac MR sequences of a rat. We compare the epicardial and the left and right venticular contours obtained by STACS with the corresponding contours manually segmented by an expert. We compare them quantitatively using two similarity measures: the area similarity, which is widely used, and the shape similarly, which we propose here for more accurate measurement.

\section{ENERGY FUNCTIONAL}

Given a cardiac MR image, our goal is to develop a method that automatically locates a contour $\mathbf{C}$ that separates the pixels of the image into two groups: the heart and the background. The method is motivated by the following four objectives.

- Model Matching: we assume that the intensities of the pixels inside and outside the heart follow different stochastic models. For pixels belonging to the heart, their corresponding intensities are modeled by a stochastic model, say $\mathcal{M}_{1}$. For pixels belonging to the background, the corresponding intensity values are described by another stochastic model $\mathcal{M}_{2}$. A similar approach is adopted when segmenting the heart structures: the left and right ventricles and the myocardium.

- Edge Information: the contour should be attracted to clues given by prominent edges in the edge map of the image.

- Shape Priors: the heart has a nominal contour. We choose to describe it by a generic shape $\mathbf{C}_{\mathrm{H}}(\theta)$ parameterized by the parameter vector $\boldsymbol{\theta}=\left[\theta_{1}, \ldots, \theta_{n}\right]^{T}$. The values of these parameters $\theta_{i}$ may determine the detailed size and shape of the actual heart and are estimated from the MR image.

- Contour Smoothness: the contour of the segmented heart should be smooth, not jagged, or too noisy.

We translate these four requirements into an objective functional $J(\mathbf{C})$ with four terms

$$
J(\mathbf{C})=\lambda_{1} J_{1}(\mathbf{C})+\lambda_{2} J_{2}(\mathbf{C})+\lambda_{3} J_{3}(\mathbf{C})+\lambda_{4} J_{4}(\mathbf{C})
$$

where $J_{1}(\mathbf{C})$ incorporates the model matching requirement (region-based); $J_{2}(\mathbf{C})$ is the edge-based information; $J_{3}(\mathbf{C})$ incorporates the prior knowledge on the shape of the contour; $J_{4}(\mathbf{C})$ regulates the smoothness of the contour; and $\lambda_{1}, \lambda_{2}, \lambda_{3}$, and $\lambda_{4}$ are parameters that control the relative strength of each of the terms $J_{1}, J_{2}, J_{3}$, and $J_{4}$, respectively.

With the contour $\mathbf{C}$ embedded as the zero of the level set function $\phi(x, y)$

$$
\mathbf{C}=\{(x, y) \in \Omega: \phi(x, y)=0\}
$$

the energy functional (1) becomes

$$
J(\phi)=\lambda_{1} J_{1}(\phi)+\lambda_{2} J_{2}(\phi)+\lambda_{3} J_{3}(\phi)+\lambda_{4} J_{4}(\phi) .
$$

We detail now each of these terms.

\section{A. Region-Based Term: Model Matching $J_{1}(\phi)$}

The goal is to segment the domain $\Omega$ of a given image into two "homogeneous" regions separated by the contour $\mathbf{C}$ : the region inside the contour, $\Omega_{1}$, represents the object region; the region outside the contour, $\Omega_{2}$, corresponds to the background region. A homogeneous region is a region whose intensity is well described by the same statistical model, or equivalently, the same probability distribution. We consider two stochastic models: the model $\mathcal{M}_{1}$ describes the statistics of the object and the model $\mathcal{M}_{2}$ represents the statistics of the background. The segmentation task partitions the image pixels $\{(i, j) \in \Omega\}$ into two regions, separated by the contour $\mathbf{C}$. Let $\Omega_{1}$ and $\Omega_{2}$ represent the regions inside and outside $\mathbf{C}$, respectively. If the pixels belong to the object, then the corresponding brightness values $\mathbf{u}_{1}=\left\{u_{i j}:(i, j) \in \Omega_{1}\right\}$ are described by the statistical model $\mathcal{M}_{1}$. On the other hand, if the pixels belong to the background, the corresponding brightness values $\mathbf{u}_{2}=\left\{u_{i j}:(i, j) \in \Omega_{2}\right\}$ are described by the statistical model $\mathcal{M}_{2}$.

Suppose that the entire image domain $\Omega$ has been initially segmented into two regions by a contour $\mathbf{C}_{0}$. To update the contour $\mathbf{C}_{0}$, we maximize the probability that $\mathbf{u}_{1}$ and $\mathbf{u}_{2}$ are random samples drawn from the object model $\mathcal{M}_{1}$ and the background model $\mathcal{M}_{2}$, respectively. To achieve this, the contour $\mathbf{C}_{0}$ changes to $\mathbf{C}_{1}$ by moving pixels from $\Omega_{1}$ to $\Omega_{2}$, and vice-versa. We control this updating from $\mathbf{C}_{0}$ to $\mathbf{C}_{1}$ by attempting to maximize the likelihood function

$$
J_{0}(\mathbf{C})=p\left(\mathbf{u} \mid \mathbf{C}, \mathcal{M}_{1}, \mathcal{M}_{2}\right)
$$

where $p\left(\mathbf{u} \mid \mathbf{C}, \mathcal{M}_{1}, \mathcal{M}_{2}\right)$ is the joint probability density function of the image intensities $\mathbf{u}$ given the contour $\mathbf{C}$ and the models $\mathcal{M}_{1}$ and $\mathcal{M}_{2}$.

Let $p_{1}$ and $p_{2}$ be the probability density functions (pdf) associated with the models $\mathcal{M}_{1}$ and $\mathcal{M}_{2}$, respectively. Since the intensities of the pixels inside and outside the contour $\mathbf{C}$ are statistically independent given the contour, (4) is equivalently rewritten as the product of the two pdfs, i.e.,

$$
J_{0}(\mathbf{C})=p_{1}\left(\mathbf{u}_{1} \mid \mathbf{C}\right) p_{2}\left(\mathbf{u}_{2} \mid \mathbf{C}\right) .
$$

Taking the negative log, (5) becomes

$$
J_{1}(\mathbf{C})=-\ln \left(p_{1}\left(\mathbf{u}_{1} \mid \mathbf{C}\right)\right)-\ln \left(p_{2}\left(\mathbf{u}_{2} \mid \mathbf{C}\right)\right),
$$

and maximizing $J_{0}(\mathbf{C})$ in (5) becomes minimizing $J_{1}(\mathbf{C})$ in (6). We assume further that the intensities of all pixels within each region are statistically independent. Therefore

$$
p_{k}\left(\mathbf{u}_{k} \mid \mathbf{C}\right)=\prod_{(i, j) \in \Omega_{k}} p_{k}\left(u_{i j}\right), \quad \text { for } \quad k=1,2
$$

and (6) becomes

$$
J_{1}(\mathbf{C})=\sum_{(i, j) \in \Omega_{1}}-\ln \left(p_{1}\left(u_{i j}\right)\right)+\sum_{(i, j) \in \Omega_{2}}-\ln \left(p_{2}\left(u_{i j}\right)\right) .
$$


When the contour $\mathbf{C}$ is embedded as the zero level of the level set function $\phi,(8)$ becomes $^{1}$

$$
\begin{aligned}
J_{1}(\phi)=\int_{\Omega}- & \ln \left[p_{1}(u(x, y))\right] \mathcal{H}_{\epsilon}(\phi(x, y)) \\
& -\ln \left[p_{2}(u(x, y))\right]\left[1-\mathcal{H}_{\epsilon}(\phi(x, y))\right] d x d y
\end{aligned}
$$

where

$$
\mathcal{H}_{\epsilon}(\phi)=\frac{1}{2}\left[1+\frac{2}{\pi} \arctan \left(\frac{\phi}{\epsilon}\right)\right]
$$

is a regularized Heaviside function representing the pixels within the contour, $1-\mathcal{H}_{\epsilon}(\phi(x, y))$ is the function representing the pixels outside the contour, and the integral is taken over the entire domain $\Omega$ of the image. This is the generic form of the first term to be included in our energy functional, and $p_{1}$ and $p_{2}$ are the assumed pdfs of the object and background models, respectively. We assume that both the object and the background pdfs are Gaussian with means $m_{1}$ and $m_{2}$, and variances $\sigma_{1}^{2}$ and $\sigma_{2}^{2}$, which are unknown and will be estimated jointly with the contour.

\section{B. Edge-Based Term $J_{2}(\phi)$}

Analogous to classical snakes [4], we want the contour to be attracted to prominent edges in the image. We realize this by minimizing the edge map $\Upsilon(x, y)$, which is derived from the original image $u(x, y)$, along the contour $\mathbf{C}$

$$
J_{2}(\mathbf{C})=\int_{\mathbf{C}} \Upsilon(x, y) d s
$$

where $d s$ represents the infinitesimal Euclidean arc length of the contour $\mathbf{C}$. The simplest edge map, which is commonly used in several edge-based active contour schemes [4]-[6], [13], is the magnitude of the gradient of the image $u(x, y)$, i.e.,

$$
\Upsilon(x, y)=-\left|\nabla G_{\sigma} * u(x, y)\right|
$$

where $G_{\sigma}$ is the 2-D Gaussian kernel with variance $\sigma^{2}, \nabla$ is the gradient operator, and $*$ is the 2-D convolution operator. The 2-D Gaussian kernel is used to smooth out the noise, thus helping to eliminate spurious edges that may occur after applying the gradient. However, this also blurs the image. Other image smoothing methods may be used, including edge-preserved anisotropic diffusions [14]-[17], the fourth order PDE method [18], or the Min/Max flow algorithm [19].

When $\mathbf{C}$ is embedded as the zero level set of $\phi,(11)$ becomes

$$
\begin{aligned}
J_{2}(\phi) & =\int_{\Omega} \Upsilon(x, y)\left|\nabla \mathcal{H}_{\epsilon}(\phi(x, y))\right| d x d y \\
& =\int_{\Omega} \Upsilon(x, y) \delta_{\epsilon}(\phi(x, y))|\nabla \phi(x, y)| d x d y
\end{aligned}
$$

where the regularized delta function

$$
\delta_{\epsilon}(\phi)=\frac{d}{d \phi} \mathcal{H}_{\epsilon}(\phi)
$$

defines the pixels that are on the contour $\mathbf{C}$.

\footnotetext{
${ }^{1}$ For notation commodity, we now assume a continuous-domain image $u(x, y)$ instead of the discrete-domain image $u_{i j}$ assumed in the first part of the development. Therefore, the summations are replaced by integrals.
}

\section{Heart Shape Prior Term $J_{3}(\phi)$}

Due to low contrast and similar textures between the tissues to be segmented, e.g., the myocardium and the papillary muscles, the region and edge based terms cannot by themselves segment the heart and its chambers successfully. Existing active contour methods [4], [5], [9], [7] do not use available information about the shape of the contour. In our application, there is reasonable knowledge about the shape of the heart: the myocardium resembles an elliptical shape. However crude this shape model is, we can exploit it and regulate the shape of the contour. We explain how to incorporate a parametrically described shape prior, as for example, an ellipse, into our active contour scheme. It is clear that more detailed models for the contour of the myocardium can be used, since the approach to be presented is general enough to cover a wide range of shapes.

An ellipse can be described by a conic equation

$$
a x^{2}+b x y+c y^{2}+d x+e y+f=0
$$

under the constraint

$$
4 a c-b^{2}>0 .
$$

Equation (16) describes the shape with six parameters. It turns out that an ellipse can be fully described with merely five parameters [20]. As a result, (16) has one extra degree of freedom, and we can arbitrarily rescale the parameters $a, b$, and $c$. The inequality constraint (17) is then equivalent to

$$
4 a c-b^{2}=\varepsilon
$$

where $\varepsilon$ is an arbitrary positive constant. If we divide the equality (18) through by $\varepsilon$, we obtain a new equality constraint

$$
4 \tilde{a} \tilde{c}-\tilde{b}^{2}=1
$$

where the new parameters $\tilde{a}, \tilde{b}$, and $\tilde{c}$ are the scaled versions of the original $a, b$, and $c$ parameters in the previous inequality constraint in (17). Collect the ellipse parameters into the vector $\boldsymbol{\theta}=\left[\begin{array}{llllll}\tilde{a} & \tilde{b} & \tilde{c} & d & e & f\end{array}\right]^{T}$ and let $\mathbf{v}=\left[\begin{array}{llllll}x^{2} & x y & y^{2} & x & y & 1\end{array}\right]^{T}$. We can then rewrite the ellipse equation compactly as

$$
\boldsymbol{\theta}^{T} \mathbf{v}=0
$$

under the constraint

$$
\boldsymbol{\theta}^{T} \mathbf{K} \boldsymbol{\theta}=1
$$

where

$$
\mathbf{K}=\left[\begin{array}{cccccc}
0 & 0 & 2 & 0 & 0 & 0 \\
0 & -1 & 0 & 0 & 0 & 0 \\
2 & 0 & 0 & 0 & 0 & 0 \\
0 & 0 & 0 & 0 & 0 & 0 \\
0 & 0 & 0 & 0 & 0 & 0 \\
0 & 0 & 0 & 0 & 0 & 0
\end{array}\right]
$$

Forcing the shape of the evolving contour $\mathbf{C}$ to resemble the ellipse contour $\mathbf{C}_{H}(\boldsymbol{\theta})$, where

$$
\mathbf{C}_{H}(\boldsymbol{\theta})=\left\{(x, y): \boldsymbol{\theta}^{T} \mathbf{v}=0 \quad \text { and } \quad \boldsymbol{\theta}^{T} \mathbf{K} \boldsymbol{\theta}=1\right\}
$$


is captured by minimizing the squared distance to the ellipse contour $\mathbf{C}_{H}(\boldsymbol{\theta})$ of the pixels on the contour $\mathbf{C}$. In other words, we minimize

$$
J_{3}(\mathbf{C})=\int_{\mathbf{C}} \mathcal{D}^{2}(x, y) d s
$$

where $\mathcal{D}(x, y)$ is the ellipse distance function defined as

$$
\mathcal{D}(x, y)=\tilde{a} x^{2}+\tilde{b} x y+\tilde{c} y^{2}+d x+e y+f .
$$

Embedding $\mathbf{C}$ as the zero of the level set function $\phi(x, y)$, we have

$$
J_{3}(\phi)=\int_{\Omega} \mathcal{D}^{2}(x, y) \delta_{\epsilon}(\phi(x, y))|\nabla \phi(x, y)| d x d y
$$

where $\mathcal{D}(x, y)$ is another level set function, representing the distance to the ellipse contour $\mathbf{C}_{H}(\boldsymbol{\theta})$, and $\delta_{\epsilon}(\phi)$ is the regularized delta function that selectively masks out only the pixels on the contour $\mathbf{C}$. The parameters $\tilde{a}, \tilde{b}, \tilde{c}, d, e$, and $f$ of the ellipse are unknown and so they are jointly estimated with the statistical model parameters and the contour itself. We note that other shape priors can be similarly incorporated-we need only define an appropriate distance between the desired shape and the current shape of the contour. Possible distances are the similarity measures introduced in Section VI-B.

\section{Contour Smoothing Term $J_{4}(\phi)$}

Lastly, we want the contour of the segmented heart to be smooth, or not too noisy. We achieve this by minimizing the total Euclidean arc length of the contour C [21], [9], [22]. In other words, we minimize

$$
J_{4}(\mathbf{C})=\int_{\mathbf{C}} d s
$$

where $d s$ represents the infinitesimal Euclidean arc length of the contour $\mathbf{C}$. If we minimize $J_{4}(\mathbf{C})$ alone, the contour $\mathbf{C}$ will evolve to become a circle and eventually shrink, to wither away and disappear. However, when we simultaneously minimize $J_{4}(\mathbf{C})$ along with the other terms in (1), the conjugate effect of all the terms prevents this, and the effect of the $J_{4}(\mathbf{C})$ term will be to force the contour to be smooth. Equation (27) can be rewritten in terms of the level set function $\phi(x, y)$ as

$$
J_{4}(\phi)=\int_{\Omega} \delta_{\epsilon}(\phi(x, y))|\nabla \phi(x, y)| d x d y
$$

where $\delta_{\epsilon}(\phi)$ is the regularized delta function.

\section{E. Summary: Energy Functional}

Replacing (9), (14), (26), and (28) in (3), we have STACS's energy functional. STACS searches for a contour $\mathbf{C}$, which is embedded as the zero level of the level set function $\phi$, that minimizes the functional

$$
\begin{aligned}
J(\phi)= & \lambda_{1} J_{1}(\phi)+\lambda_{2} J_{2}(\phi)+\lambda_{3} J_{3}(\phi)+\lambda_{4} J_{4}(\phi) \\
= & \int_{\Omega} \lambda_{1} M_{1} \mathcal{H}_{\epsilon}(\phi(x, y))+\lambda_{1} M_{2}\left[1-\mathcal{H}_{\epsilon}(\phi(x, y))\right] \\
& +P(x, y) \delta_{\epsilon}(\phi(x, y))|\nabla \phi(x, y)| d x d y
\end{aligned}
$$

where

$$
M_{k}=\frac{1}{2} \ln \left(2 \pi \sigma_{k}^{2}\right)+\frac{\left(u(x, y)-m_{k}\right)^{2}}{2 \sigma_{k}^{2}} \quad \text { for } \quad k=1,2
$$

are the negative log of the pdfs of the object and the background models, respectively; and the potential function

$$
P(x, y)=\lambda_{2} \Upsilon(x, y)+\lambda_{3} \mathcal{D}^{2}(x, y)+\lambda_{4}
$$

where $\Upsilon$ is the edge map given by (12), and $\mathcal{D}$ the ellipse distance function given by (25). The parameters $\lambda_{1}, \lambda_{2}, \lambda_{3}$, and $\lambda_{4}$ control the relative strength of $J_{1}(\phi), J_{2}(\phi), J_{3}(\phi)$, and $J_{4}(\phi)$, respectively. The means and variances for the object and background models, $m_{1}, \sigma_{1}^{2}, m_{2}$, and $\sigma_{2}^{2}$, are yet to be determined. The edge map $\Upsilon(x, y)$ is static and it is derived only once from the image $u(x, y)$. The ellipse distance $\mathcal{D}(x, y)$, on the other hand, is dynamic and involves the unknown set of parameters in the parameter vector $\boldsymbol{\theta}$ that defines the ellipse shape.

\section{ENERGy FunCtional MinimizATION}

We minimize the functional (29) by iterating between three tasks. In the first and second tasks, we fix the contour $\mathbf{C}$, and then, first, estimate the parameters $m_{k}$ and $\sigma_{k}^{2}$ for $k=1,2$ of both models in $J_{1}(\phi)$ and, second, estimate the parameters $\boldsymbol{\theta}$ of the ellipse contour in $J_{3}(\phi)$. In the third task, we fix all these parameters, and then evolve the contour $\mathbf{C}$, or equivalently the level set function $\phi$, so that the functional (29) is minimized. We explain each of the three tasks in the following paragraphs, respectively.

Task 1) Estimation of the Stochastic Model Parameters: The parameters needed to be estimated for the object and background Gaussian models are the means $m_{k}$ and the variances $\sigma_{k}^{2}$, for $k=1,2$. Finding these parameters is straightforward. Fixing $\phi$, we take the derivatives of $J$ in (29) with respect to these parameters and equate the resulting expressions to zero, then solve for the parameters. As a result, the estimated means and variances are simply the sample means and variances corresponding to the pixels inside and outside the contour $\mathbf{C}$, respectively. Given the contour $\mathbf{C}$, they are the maximum likelihood estimates of the means and variances of the statistical models $\mathcal{M}_{1}$ and $\mathcal{M}_{2}$.

Task 2) Estimation of the Ellipse Parameters: We describe how to estimate the ellipse parameter vector $\boldsymbol{\theta}=$ $\left[\begin{array}{lllll}\tilde{a} & \tilde{b} & \tilde{c} & d & e\end{array}\right]^{T}$ using least squares, [20]. Given a set of coordinates $\left\{\left(x_{k}, y_{k}\right): 1 \leq k \leq n\right\}$ where $n$ is the total number of pixels on the contour $\mathbf{C}$,

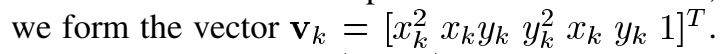
The ellipse distance $\mathcal{D}\left(x_{k}, y_{k}\right)$, the distance from coordinate $\left(x_{k}, y_{k}\right)$ to the ellipse parameterized by $\boldsymbol{\theta}$, is compactly written as $\boldsymbol{\theta}^{T} \mathbf{v}_{k}$, see (20). The sum of the squared ellipse distances is

$$
\sum_{k=1}^{n}\left(\boldsymbol{\theta}^{T} \mathbf{v}_{k}\right)^{2}=\boldsymbol{\theta}^{T} \mathbf{R} \boldsymbol{\theta}
$$


with

$$
\mathbf{R}=\sum_{k=1}^{n} \mathbf{v}_{k} \mathbf{v}_{k}^{T}=\mathbf{V} \mathbf{V}^{T}
$$

where $\mathbf{V}=\left[\begin{array}{llll}\mathbf{v}_{1} & \mathbf{v}_{2} & \cdots & \mathbf{v}_{n}\end{array}\right]$.

Our goal is to find the ellipse parameter vector $\boldsymbol{\theta}$ that minimizes the sum of the squared distances $\boldsymbol{\theta}^{T} \mathbf{R} \boldsymbol{\theta}$, subject to the constraint $\boldsymbol{\theta}^{T} \mathbf{K} \boldsymbol{\theta}=1$. Introducing the Lagrange multiplier $\mu$ and taking the derivative with respect to $\boldsymbol{\theta}$, we have the generalized eigenvalue problem, [23]

$$
\begin{aligned}
\mathbf{R} \boldsymbol{\theta} & =\mu \mathbf{K} \boldsymbol{\theta}, \\
\boldsymbol{\theta}^{T} \mathbf{K} \boldsymbol{\theta} & =1 .
\end{aligned}
$$

If an eigenvalue-eigenvector pair $\left(\mu_{i}, \mathbf{w}_{i}\right)$ solves (34), so does $\left(\mu_{i}, \rho \mathbf{w}_{i}\right)$ for any constant $\rho$. If we let the solution be $\hat{\boldsymbol{\theta}}=\rho \mathbf{w}_{i}$ and substitute it in the constraint (35), we have $\rho^{2} \mathbf{w}_{i}^{T} \mathbf{K} \mathbf{w}_{i}=1$ or

$$
\rho_{i}=\sqrt{\frac{1}{\mathbf{w}_{i}^{T} \mathbf{K} \mathbf{w}_{i}}}=\sqrt{\frac{\mu_{i}}{\mathbf{w}_{i}^{T} \mathbf{R} \mathbf{w}_{i}}} .
$$

Although there can be up to six eigensolutions, $\rho_{i}$ is only valid if the quantity under the square root is positive. Since $\mathbf{R}$ is nonnegative definite and generally positive definite, $\rho_{i}$ is only valid when $\mu_{i}$ is positive. It has been proven in [20] that there is exactly one solution to (34) under the constraint (35) that corresponds to the single positive generalized eigenvalue of (34) and (35).

Task 3) Contour Evolution Equation: In the third task, assuming that all parameters are known, we evolve the level set function $\phi$, hence the contour $\mathbf{C}$, so that it minimizes the functional (29). In Appendix I, we present the Euler-Lagrange equation corresponding to the functional (29) as

$$
\left[\lambda_{1}\left(M_{1}-M_{2}\right)-\nabla P \cdot \frac{\nabla \phi}{\|\nabla \phi\|}-P \kappa\right] \delta_{\epsilon}(\phi)=0
$$

where

$$
\kappa=\operatorname{div}\left(\frac{\nabla \phi}{\|\nabla \phi\|}\right)
$$

represents the curvature of the contour, and the potential force field

$$
\nabla P=\lambda_{2} \nabla \Upsilon+2 \lambda_{3} \mathcal{D} \nabla \mathcal{D} \text {. }
$$

We solve for $\phi$ in (37) iteratively, using the gradient descent method, by letting $\phi$ be a function of time and replace the zero on the right-hand side of (37) by the time derivative of $\phi$. Thus, we obtain the PDE

$$
\frac{\partial \phi}{\partial t}=\left[\lambda_{1}\left(M_{1}-M_{2}\right)-\nabla P \cdot \frac{\nabla \phi}{\|\nabla \phi\|}-P \kappa\right] \delta_{\epsilon}(\phi) .
$$

\section{Stochastic RelaXation: $\lambda$-ANNEALING}

When segmenting the heart in MR image sequences by minimizing the energy functional (1), which is a linear combination of the four energy terms, $J_{1}$ to $J_{4}$ with $\lambda_{1}$ to $\lambda_{4}$ as their weighting parameters, respectively, we encounter an important issue: how to choose the appropriate values for the weighting parameters $\lambda_{1}$ to $\lambda_{4}$. Their relative values emphasize differently each of the energy terms $J_{1}$ to $J_{4}$ in the functional. For instance, if one parameter $\lambda_{i}$ is much larger than the others, it is expected that the corresponding term $J_{i}$ will guide the segmentation. This, of course, has major implications in the final results. In practice, choosing these parameters is usually done empirically based on the cumulative experience of the designer, as developed after testing the minimization algorithm with many different data. In our experience with the heart MR image sequences, there is no universally fixed good set of values for these parameters. Our experience has also shown that in fact the values of these $\lambda_{i}$ 's should change as the contour evolves, meaning that the functional itself should evolve over time. Intuitively, when the segmentation is started, the region and edge-based terms, $J_{1}$ and $J_{2}$, should be the main drivers of the minimization process, forcing the initial contour to evolve close to relevant boundaries. This is because these two terms are image dependent, i.e., derived directly from the image, and it is important that the contour evolves freely following the local image conditions. At later stages in the minimization process, the shape prior becomes an important feature because eventually we want the contour to resemble the assumed shape prior, or at least not be dramatically different. Therefore, the weights $\lambda_{i}$ 's should adapt and evolve as the segmentation proceeds.

We present here an annealing algorithm for the parameters $\lambda_{i}$ 's that adapt their values as the segmentation evolves: initially emphasizing the terms $J_{1}$ and $J_{2}$ with large values of $\lambda_{1}$ and $\lambda_{2}$, and, at later stages, decreasing their values while increasing the importance of the shape prior term $J_{3}$ through $\lambda_{3}$. The algorithm gradually adapts the weighting parameters $\lambda_{1}, \lambda_{2}$, and $\lambda_{3}$. We choose the following annealing schedules

$$
\begin{aligned}
& \lambda_{1}(n)=\lambda_{1}(1)-\frac{n\left[\lambda_{1}(1)-\lambda_{1}(N)\right]}{N} \\
& \lambda_{2}(n)=\frac{1}{2}\left[\lambda_{2}(1)-\lambda_{2}(N)\right]\left[1+\cos \left(\frac{n \pi}{N}\right)\right]+\lambda_{2}(N) \\
& \lambda_{3}(n)=\frac{\lambda_{3}(N)-\lambda_{3}(1)}{\cosh \left[10\left(\frac{n \pi}{N}-1\right)\right]}+\lambda_{3}(1)
\end{aligned}
$$

where $n$ is the iteration number, $N$ is the total number of iterations, and $\lambda_{i}(1)$ and $\lambda_{i}(N)$ are the initial and final values of $\lambda_{i}$, respectively. The annealing schedule for $\lambda_{1}$ is linear, a common choice in many applications. The annealing schedule for $\lambda_{2}$ updates the value moderately in the middle range according to a cosine function. The annealing schedule for $\lambda_{3}$ increases from the initial value $\lambda_{3}(1)$ to the final value $\lambda_{3}(N)$ most dramatically at the end of the process. We choose $\lambda_{4}$ to be constant throughout the minimization process.

We stop the segmentation process by fixing the maximum number $N$ of iterations. There are other possible stopping criteria, for example, by controlling the relative change of the contour and stopping when this change is below a threshold. To measure the relative changes of the contour, we may use the shape similarity measure introduced in Section VI-B. This makes each iteration computationally more complex. By adopting our simple criterion, we can choose a larger number of iterations, which gives us confidence, confirmed by the experimental results in Section VI, that the contour has stabilized. In our experiments, we used a fixed maximum number of iterations $N=200$; and took the initial and final values 


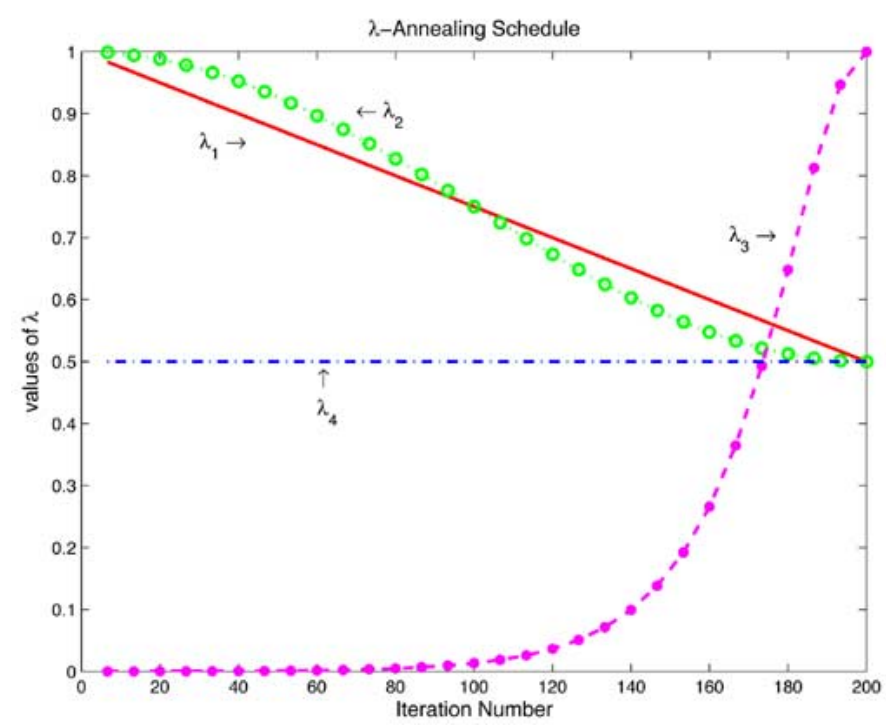

Fig. 1. $\lambda$-annealing schedule.

for $\lambda_{1}$ and $\lambda_{2}$ to be, respectively, $\lambda_{1}(1)=\lambda_{2}(1)=1$ and $\lambda_{1}(N)=\lambda_{2}(N)=0.5$. The initial and final values for $\lambda_{3}$ are 0 and 1 , respectively. The constant value for $\lambda_{4}$ is 0.5 . Fig. 1 depicts the plots of the $\lambda$-annealing schedule that we use. Our STACS algorithm nests two loops: the outer loop sets each $\lambda_{i}$ according to the corresponding annealing schedules above, and the inner loop minimizes the energy functional (29) by iterating between the three tasks described in Section III.

\section{Data Aquisition}

The cardiac MR data that we used were acquired by a Brucker AVANCE DRX 4.7 tesla system from the Pittsburgh NMR Center for Biomedical Research at the CMU Mellon Institute. An electrocardiogram and respiration-gated spin-echo cine imaging was used to obtain high-resolution density-weighted short axis images with:

- $4 \mathrm{~cm}$ field of view;

- $156 \mathrm{~mm}$ by $156 \mathrm{~mm}$ in-plane resolution;

- $1.5 \mathrm{~mm}$ slice thickness;

- one cardiac cycle (about $165 \mathrm{~ms}$ ) repetition time;

- and $5.5 \mathrm{~ms}$ echo time.

\section{RESULTS}

\section{A. Comparison With Other Active Contour Methods}

We compare the STACS with other active contour algorithms when applied to a real cardiac MR image. Fig. 2(a)-(c) depicts the segmentation results obtained with the Xu and Prince's GVF snake [7], the Chan and Vese's active contour algorithm [9], and our STACS, respectively. The same initial contours, indicated in dashed blue lines, are used in the three methods. The final contours are shown in solid red lines. Lastly, Fig. 2(d) shows the left ventricular endocardium contour traced manually by a trained specialist. We see that the lower part of the contour in the GVF snake algorithm, which is purely edge-based, fails to capture the bottom part of the left ventricular endocardium. It evolved out to track the boundary of the epicardium instead. This is because the edge information at the bottom part of the left ventricular endocardium is too weak and the initial contour is located a little too far from the endocardium. The result in Fig. 2(b) with the Chan and Vese's algorithm, which assumes piecewise constant models, can delineate the whole boundary of the left ventricular endocardium, but fails to separate the papillary muscles out of the left ventricle. The result when applying STACS in Fig. 2(c) shows that the papillary muscles are correctly segmented. The shape priors play a significant role in segmenting the papillary muscles out of the left ventricle. Among these three segmentation results of the left ventricular endocardium by different algorithms, the result from STACS in Fig. 2(c) shows most resemblence to the contour traced manually by an expert shown in Fig. 2(d).

\section{B. Quality Assessment}

Fig. 3(a) and (b) shows the segmentation results obtained by STACS and the contours traced manually by an expert on the same set of 48 cardiac MR images, respectively. Three sets of contours, representing the boundaries of the left ventricle (LV), the right ventricle (RV), and the epicardium (EP) of a rat heart are present (see Section V for details on the MR data). Each set of contours was obtained separately by STACS. First, we place a bounding box around the rat heart in each frame of the cardiac MR image set. This is done automatically by detecting the motion of the heart through the cycle. Second, we determine the centers of the left and right ventricles by searching for the two peaks (one for the LV, the other for the RV) in the horizontal projection of the image intensity. Details are provided in [24]. We then place, one at a time, two circles centered at these two points as the initial contours for segmenting the left and right ventricles. Once the left and right ventricles are segmented out, a ellipse is placed as an initial contour to detect the epicardium.

Our goal is to find a quantitative method that compares our automatically generated contours in Fig. 3(a) with the manually traced "gold standard" contours in Fig. 3(b). We introduce two similarity measures: the area similarity and the shape similarity. We explain the methodology of each of them in the following subsections, respectively.

1) Area Similarity: One of the methods commonly used for assessing the validation of functional MR image segmentation is to compare the area of the segmented object in the image against the one in a reference image [25], [26]. Consider two binary images $A_{1}$ and $A_{2}$ whose "on" pixels represent the regions of the segmented object. The area similarity measure, $S_{\text {area }} \in[0,1]$, between the two segmentations $A_{1}$ and $A_{2}$ is defined as the ratio between twice their common area over the sum of the individual area

$$
S_{\text {area }}=\frac{2 n\left(A_{1} \wedge A_{2}\right)}{n\left(A_{1}\right)+n\left(A_{2}\right)}
$$

where $\wedge$ is the element-wise "and" operator, and $n(A)$ represents the cardinality of $A$ or the number of "on" pixels in the binary image $A$. According to [25], $S_{\text {area }}>0.70$ indicates an excellent agreement between the two comparing regions. We computed this area similarity measure for the segmented LV, $\mathrm{RV}$, and EP contours. We summarize the numbers in three matrices, one for each contour pairs. Entry $(i, j)$ in each of these matrices is the appropriate area similarity measure for the $(i, j)$ image pair in Fig. 3(a) and (b). We were able to compute the similarity measure for every pair of corresponding images in Fig. 3(a) and (b), except for the LV contour in the $(1,3)$ image 


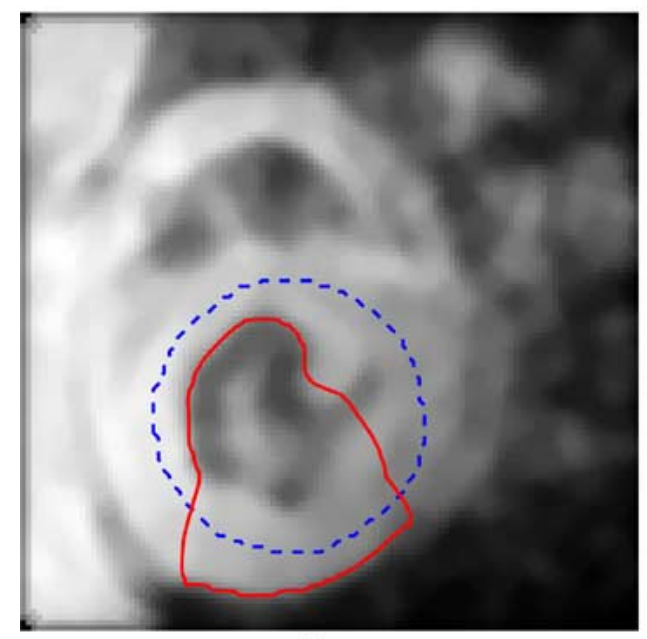

(a)

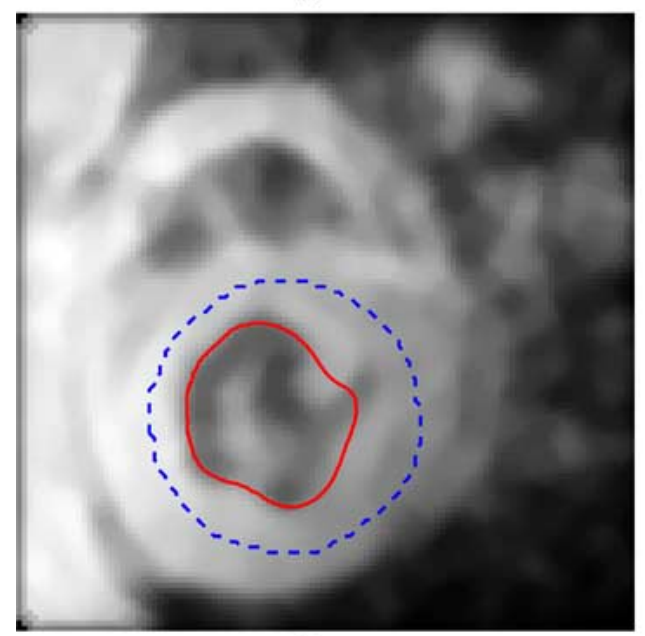

(c)

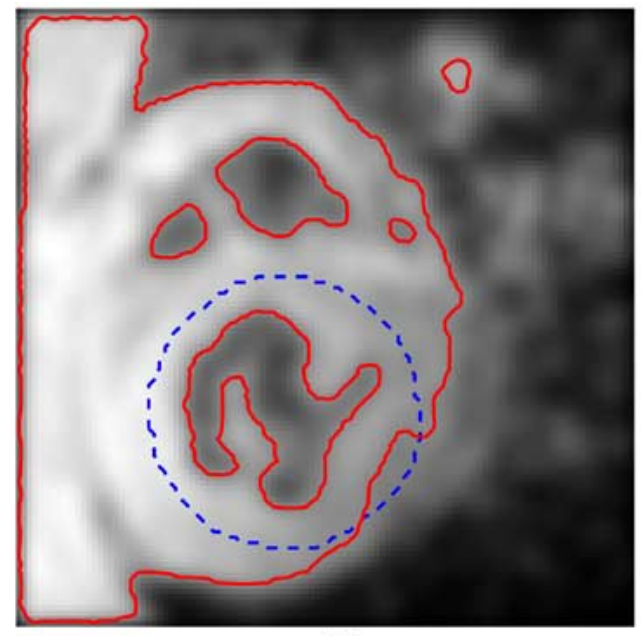

(b)

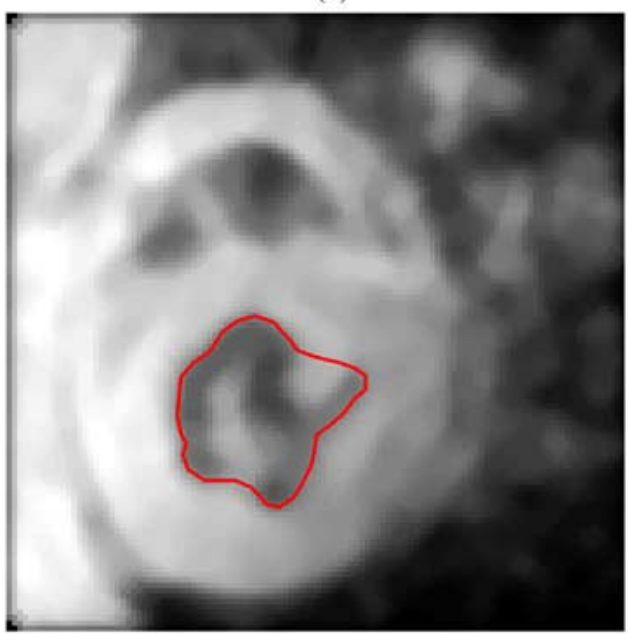

(d)

Fig. 2. Comparing the segmentation results of a cardiac MR image using different algorithms. Initial contours are the dashed blue lines; the final contours are the solid red lines. In (a), Xu and Prince's GVF snake captures only the upper part of the LV boundary, the bottom part of the contour converges to the epicardium instead of the endocardium of the left ventricle. In (b), Chan and Vese's active contour algorithm is used; the LV contour in (b) does not segment the papillary muscles because there is no information about the global shape of the left ventricle. In (c), STACS segments correctly the left ventricle; it solves the difficult segmentation of the papillary muscles by using the prior shape of the left ventricle. Among these three LV segmentation results by different algorithms, the result from STACS in (c) is most similar to the contour traced manually by an expert shown in (d). (a) Xu and Prince's GVF snake algorithm. (b) Chan and Vese's active contour algorithm. (c) STACS. (d) Contour traced manually by an expert.

in Fig. 3(a) where the left ventricle appears to be closed completely. The area similarity matrices for the left ventricle, right ventricle, and the epicardium are, respectively $S_{\text {area }}^{\mathrm{LV}}=$

$\left[\begin{array}{llllllll}0.92 & 0.85 & & 0.68 & 0.86 & 0.71 & 0.90 & 0.93 \\ 0.94 & 0.91 & 0.92 & 0.92 & 0.90 & 0.96 & 0.91 & 0.86 \\ 0.87 & 0.93 & 0.94 & 0.87 & 0.95 & 0.91 & 0.90 & 0.88 \\ 0.94 & 0.74 & 0.85 & 0.77 & 0.85 & 0.94 & 0.89 & 0.92 \\ 0.94 & 0.85 & 0.85 & 0.90 & 0.91 & 0.95 & 0.86 & 0.93 \\ 0.89 & 0.87 & 0.94 & 0.83 & 0.94 & 0.85 & 0.82 & 0.78\end{array}\right]$

$S_{\text {area }}^{\mathrm{RV}}=$

$\left[\begin{array}{llllllll}0.84 & 0.64 & 0.85 & 0.73 & 0.88 & 0.80 & 0.90 & 0.83 \\ 0.89 & 0.83 & 0.75 & 0.86 & 0.73 & 0.90 & 0.91 & 0.90 \\ 0.86 & 0.63 & 0.83 & 0.80 & 0.78 & 0.92 & 0.92 & 0.86 \\ 0.81 & 0.65 & 0.56 & 0.67 & 0.85 & 0.93 & 0.84 & 0.91 \\ 0.91 & 0.75 & 0.74 & 0.82 & 0.79 & 0.93 & 0.87 & 0.90 \\ 0.82 & 0.80 & 0.65 & 0.70 & 0.79 & 0.89 & 0.93 & 0.84\end{array}\right]$

(45)

(46)

$$
\begin{aligned}
& S_{\text {area }}^{\mathrm{EP}}= \\
& {\left[\begin{array}{lllllllll}
0.96 & 0.95 & 0.94 & 0.88 & 0.95 & 0.92 & 0.90 & 0.92 \\
0.97 & 0.96 & 0.97 & 0.91 & 0.94 & 0.96 & 0.95 & 0.96 \\
0.95 & 0.95 & 0.95 & 0.97 & 0.96 & 0.95 & 0.97 & 0.96 \\
0.97 & 0.97 & 0.95 & 0.97 & 0.95 & 0.96 & 0.93 & 0.93 \\
0.97 & 0.97 & 0.97 & 0.96 & 0.97 & 0.96 & 0.97 & 0.96 \\
0.95 & 0.95 & 0.96 & 0.96 & 0.96 & 0.96 & 0.96 & 0.96
\end{array}\right] .}
\end{aligned}
$$

We observe that, except for the $(4,1)$ position, values of the area similarity measure for the left ventricles have values greater than 0.70 . For the right ventricles, we find 41 out of the 48 frames having area similarity values greater than 0.70 , while the remaining 7 values are moderately below (or equal to) 0.70 . We also observe that turbulent blood flows appear in each of the frames with area similarity below 0.70 . This turbulent blood flow may be the cause for the less satisfactory performance of the segmentation of the right ventricle with these images. Finally, all of the epicardium area similarity values are above 0.88 , which indicates excellent results in agreement with the gold standard. 


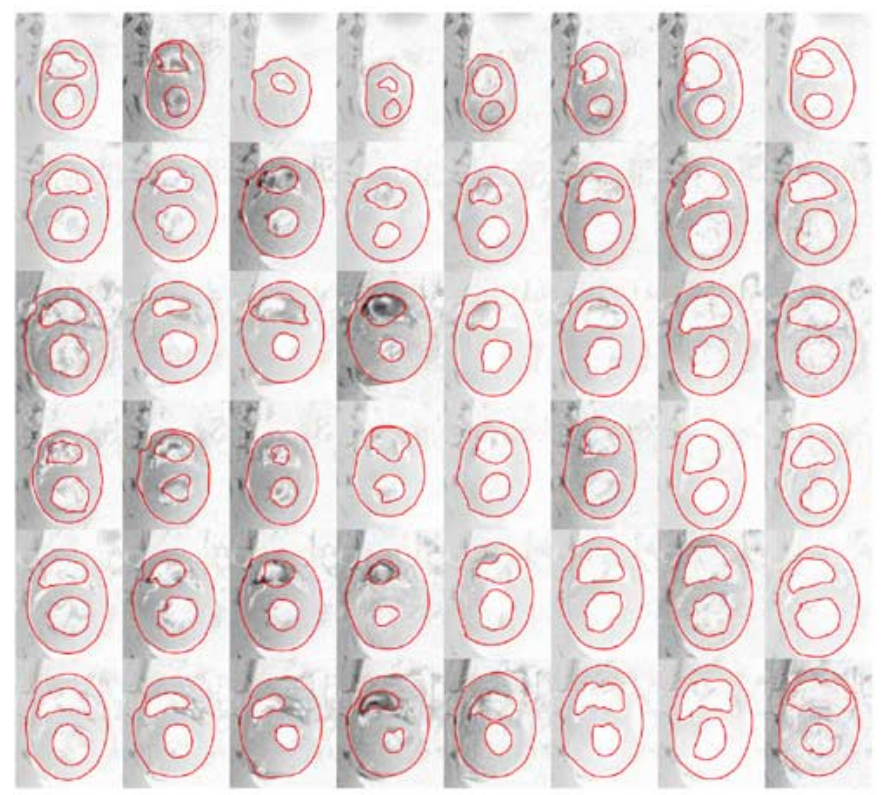

(a)

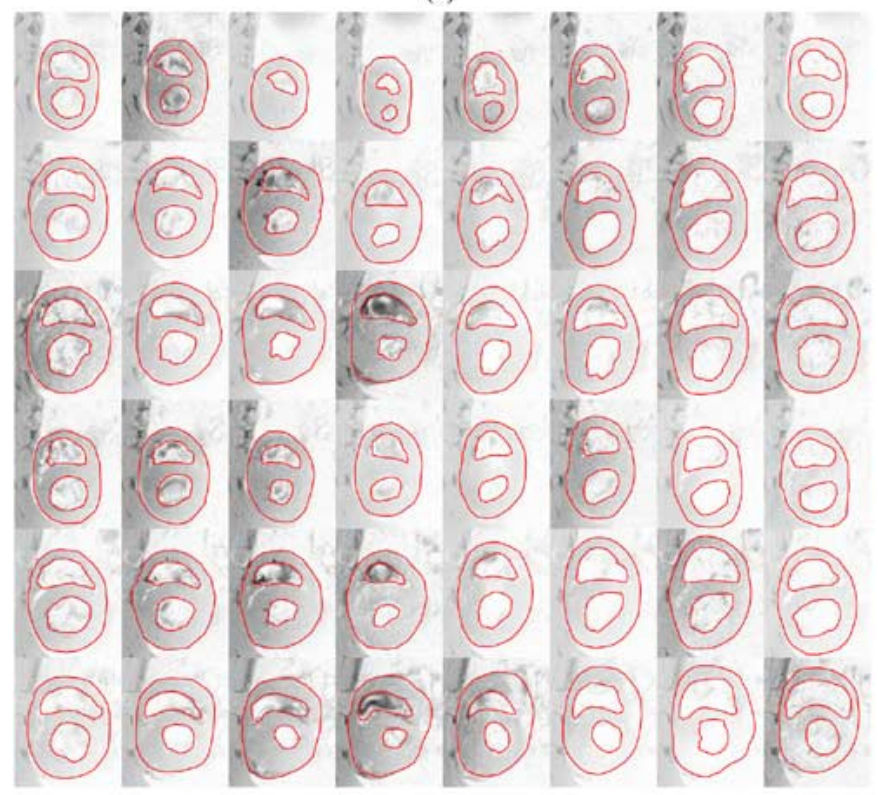

(b)

Fig. 3. Comparing the segmentation results obtained automatically by STACS in (a) with the "gold standard" hand-traced results in (b): six rat cardiac sequences of eight frames each throughout one cardiac cycle, and three contours in each image are the left and right ventricles, and the epicardium. (a) Contours obtained automatically by STACS. (b) Contours traced manually by an expert.

2) Shape Similarity: Although the area similarity measure (44), when comparing two contours, is a good indicator on how similar their sizes (or areas) and their relative locations are, it may be less informative with respect to details on the shapes of the two contours. A pair of very different shape contours may yield the same area similarity measure as a pair of contours with identical shape as long as their intersecting regions and the sums of individual areas inside the contour pair are the same. We discuss an alternative measure to assess the similarity in shape between a pair of contours, a modification of the chamfer matching method. Chamfer matching [27] is a technique for finding a match between two contour shapes, where the two contours are represented in a form of binary edge templates. It is originally and still often used in image and video processing to search for an object with a particular shape within an image. In the medical image processing framework, however, chamfer matching is often employed for image registration [28]. In our case, we will modify the chamfer matching method and use it to assess the shape similarity between a pair of contours. We follow the idea described in [29]-[31] where a modified chamfer measure is applied to capture human motion in live video (see [31]). We adjust the similarity measure in [29]-[31] so that our shape similarity index emphasizes the difference in shapes rather than the relative locations of the two comparing contours.

The flow chart for computing our shape similarity measure is illustrated in Fig. 4. Let $\mathbf{C}_{1}$ and $\mathbf{C}_{2}$ be two contours. The contour $\mathbf{C}_{1}$ is the set of coordinates of the reference contour, or the gold standard contour, and the contour $\mathbf{C}_{2}$ is the set of coordinates of the contour obtained automatically by STACS, which we will call the automatic contour. Our goal is to find a similarity measure $S_{\text {shape }} \in[0,1]$ that quantitatively assesses how similar the shape of the two contours $\mathbf{C}_{1}$ and $\mathbf{C}_{2}$ are. Our algorithm for the determination of the shape similarity measure proceeds in several steps. First, we generate the binary edge templates, $E_{1}$ and $E_{2}$, where the "on" pixels represent the pixels on each of the two contours being compared. Second, we propagate the shape of the contours in each binary edge template by applying the signed Euclidean distance transform

$$
\begin{aligned}
& \mathcal{D}(x, y)= \\
& \begin{cases}-\min _{(i, j) \in \mathbf{C}} \sqrt{(x-i)^{2}+(y-j)^{2}}, & \text { if }(x, y) \in \Omega_{1} \\
\min _{(i, j) \in \mathbf{C}} \sqrt{(x-i)^{2}+(y-j)^{2}}, & \text { if }(x, y) \in \Omega_{2}\end{cases}
\end{aligned}
$$

where $(x, y)$ represents the pixels in the image domain, $(i, j) \in$ $\mathrm{C}$ represents the pixels on the contour $\mathbf{C}$, and $\Omega_{1}$ and $\Omega_{2}$ are sets of pixels inside and outside contour $\mathbf{C}$, respectively. For details on how to implement the signed distance transform, see [32]. Applying the signed Euclidean distance transform (48) to the binary edge templates $E_{1}$ and $E_{2}$, we obtain the corresponding distance maps, $\mathcal{D}_{1}$ and $\mathcal{D}_{2}$, respectively. These distance maps simply contain the scaling replicas of the contour shapes, represented in different level sets, throughout the image domain. In the third step, we calculate the corresponding phase maps by taking the inverse tangent of the ratio of the gradient components in each distance map, i.e.,

$$
\Phi_{i}(x, y)=\tan ^{-1} \frac{\nabla_{y} \mathcal{D}_{i}(x, y)}{\nabla_{x} \mathcal{D}_{i}(x, y)} \quad \text { for } \quad i=1,2
$$

where $\nabla_{x} \mathcal{D}_{i}$ and $\nabla_{y} \mathcal{D}_{i}$ represent the $x$ and $y$ components of the gradient of the distance map $\mathcal{D}_{i}$, respectively. In the fourth step, we compute the normalized phase similarity between the two contours by

$$
S_{\text {phase }}=\frac{\left|\Phi_{1}-\Phi_{2}-\pi\right|}{\pi} .
$$

The index $S_{\text {phase }}$ takes values in $[0,1]$. A value of 1 for $S_{\text {phase }}$ indicates that the contours have the same phase and a value of 0 refers to the maximum phase difference of $\pi$. In the final step, we measure the shape similarity by taking the weighted sum of 


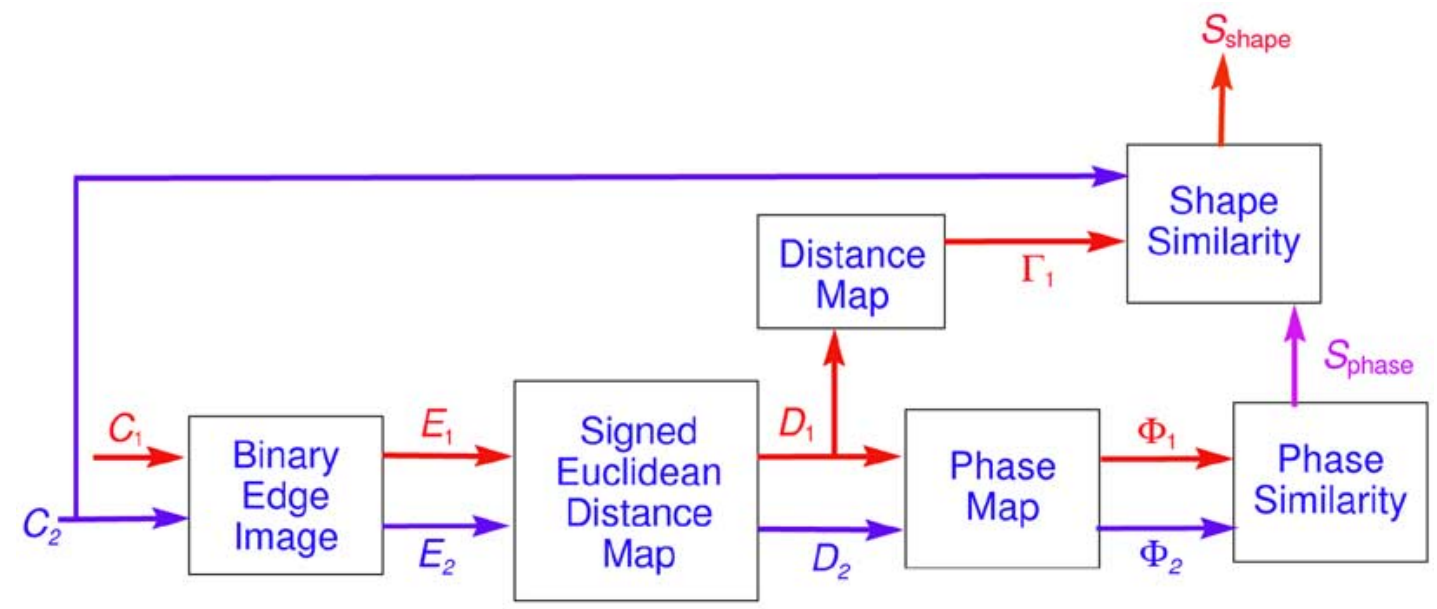

Fig. 4. Flow chart for shape similarity measure.

the phase similarity measure along $\mathbf{C}_{2}$, the automatic contour, against $\mathbf{C}_{1}$, the reference contour, i.e.,

$$
S_{\text {shape }}=\frac{1}{n\left(\mathbf{C}_{2}\right)} \sum_{(x, y) \in \mathbf{C}_{2}} \Gamma_{1}(x, y) S_{\text {phase }}(x, y)
$$

where $\mathbf{C}_{2}$ is the set of pixels on the automatic contour, $n\left(\mathbf{C}_{2}\right)$ denotes the cardinality of $\mathbf{C}_{2}$, or the number of pixels on the contour $\mathbf{C}_{2}$, and $\Gamma_{1}(x, y) \in[0,1]$ is derived from $\mathcal{D}_{1}$, the distance map of the reference contour, as

$$
\Gamma_{1}(x, y)=\exp \left\{-\frac{\mathcal{D}_{1}^{2}(x, y)}{\sigma^{2}}\right\}
$$

where $\sigma^{2}$ is a positive constant.

The findings for the quality assessment using shape similarity measure are given by the matrices

$$
\begin{aligned}
& S_{\text {shape }}^{\mathrm{LV}}= \\
& {\left[\begin{array}{llllllll}
0.90 & 0.83 & & 0.71 & 0.86 & 0.66 & 0.89 & 0.94 \\
0.91 & 0.87 & 0.89 & 0.88 & 0.85 & 0.92 & 0.84 & 0.71 \\
0.71 & 0.91 & 0.89 & 0.85 & 0.92 & 0.87 & 0.84 & 0.86 \\
0.89 & 0.70 & 0.86 & 0.75 & 0.83 & 0.92 & 0.87 & 0.83 \\
0.91 & 0.77 & 0.77 & 0.91 & 0.84 & 0.91 & 0.70 & 0.89 \\
0.80 & 0.81 & 0.92 & 0.83 & 0.92 & 0.80 & 0.68 & 0.62
\end{array}\right]}
\end{aligned}
$$

$$
\begin{aligned}
& S_{\text {shape }}^{\mathrm{RV}}= \\
& {\left[\begin{array}{llllllll}
0.77 & 0.43 & 0.86 & 0.88 & 0.84 & 0.80 & 0.84 & 0.77 \\
0.86 & 0.85 & 0.71 & 0.83 & 0.66 & 0.84 & 0.88 & 0.85 \\
0.83 & 0.68 & 0.77 & 0.71 & 0.77 & 0.87 & 0.86 & 0.79 \\
0.79 & 0.43 & 0.64 & 0.40 & 0.72 & 0.89 & 0.75 & 0.90 \\
0.88 & 0.73 & 0.70 & 0.83 & 0.65 & 0.89 & 0.78 & 0.87 \\
0.81 & 0.87 & 0.78 & 0.77 & 0.67 & 0.84 & 0.87 & 0.68
\end{array}\right]}
\end{aligned}
$$$$
S_{\text {shape }}^{\text {EP }}=
$$$$
\left[\begin{array}{llllllll}
0.92 & 0.88 & 0.85 & 0.74 & 0.90 & 0.77 & 0.61 & 0.76 \\
0.90 & 0.89 & 0.91 & 0.60 & 0.84 & 0.89 & 0.83 & 0.90 \\
0.85 & 0.84 & 0.85 & 0.88 & 0.88 & 0.83 & 0.92 & 0.84 \\
0.92 & 0.90 & 0.85 & 0.92 & 0.88 & 0.88 & 0.75 & 0.74 \\
0.92 & 0.91 & 0.91 & 0.89 & 0.90 & 0.87 & 0.89 & 0.86 \\
0.87 & 0.84 & 0.90 & 0.86 & 0.87 & 0.89 & 0.86 & 0.91
\end{array}\right] .
$$

TABLE I

Average Values of the Similarity Measures

\begin{tabular}{c|c|c|c}
\hline & LV & RV & EP \\
\hline Area Similarity & $0.88 \pm 0.06$ & $0.82 \pm 0.09$ & $0.95 \pm 0.02$ \\
\hline Shape Similarity & $0.84 \pm 0.08$ & $0.77 \pm 0.12$ & $0.86 \pm 0.07$ \\
\hline
\end{tabular}

For the left ventricles, most values of the shape similarity measure are above 0.70 . Only five contour pairs have a shape similarity measure in the $0.60-0.70$ range. We conclude that the automatic segmentation results for the left ventricles are very good. For the right ventricles, 38 out of 48 contour pairs have shape similarity values above $0.70 ; 7$ contour pairs have shape similarity values between 0.60 and 0.70 ; and 3 shape similarity values fall below 0.50. Again, in the image frames with low similarity measures, blood flow may have affected the performance of the algorithm. Finally, we find 46 out of 48 values for the epicardium shape similarity measures to be well above 0.70 , indicating excellent segmentation agreement with the gold standard.

\section{Discussion}

Table I shows the average values of all 48 similarity measures, categorized by methods and types of contours. From this table, we can conclude the following.

- Since all average values are approximately 0.8 or 0.9 and the standard deviations are quite small, we conclude that our algorithm consistently produces good results.

- We observe that the average values for both similarity measures are highest for the epicardium, and lowest for the right ventricle. This shows the highly effective influence of the shape priors that we imposed onto the heart contours. Since the epicardial boundary resembles an ellipse shape more strongly than the right ventricular boundary, our segmentation results reflect better agreement (with the hand-drawn contours) for the epicardium than the right ventricle. Further, the papillary muscles and the turbulent blood flow within the left and right ventricular regions in some of the frames may somewhat inhibit a good segmentation outcome. To improve the performance of the segmentation of the right ventricles, a better model than an ellipse, thus more complicated, may be easily incorporated into our model by adjusting the distance function $\mathcal{D}(x, y)$ in (25) in $J_{3}$. 
- As may be expected, the shape similarity measures generally yield smaller values than the area similarity measures. This is because the shape similarity measure is more sensitive to local discrepancies between the contours than the area similarity measure, which is a more global measure.

- Finally, with an overall average value of $0.88 \pm 0.09$ for the area similarity and $0.82 \pm 0.10$ for the shape similarity, we conclude that the segmentation results of STACS, in general, exhibit excellent agreement with the "gold standard" hand-traced contours.

\section{CONCLUSION}

In conclusion, we have designed, developed, and validated a new active contour scheme for cardiac MR image segmentation, called stochastic active contour scheme (STACS). The advantages of STACS are threefold. First, STACS assumes stochastic models rather than deterministic constants. As a result, it can be applied to a large range of images, particularly when the object is difficult to distinguish from the background. Second, since STACS utilizes both region-based information as well as edgebased information, the scheme is less sensitive to the contour's initial condition and more robust to noise than other purely edgebased active contour schemes. The stochastic region-based information helps propagating the contour when the initial contour is far away from the object's boundary and no edge information is present, thus increasing the capture range. Furthermore, the balancing between the region-based forces and the edge-based forces helps stabilizing the contour at the desired true boundary of the organ part we want to segment. In other words, STACS is more global, exhibits increased robustness to noise, displays extensive capture range, and is less sensitive to initial placement of the contour when compared to other active contour methods. Third, STACS conveniently incorporates the prior knowledge of the object's shape onto the global properties of the contour. This is a distinguishing feature in STACS. This shape prior that we impose onto the global properties of the contour helps overcoming the problem with the papillary muscles, whose texture is difficult to distinguish from the myocardial texture, and the chest wall in the image, that exhibits very low contrast with respect to the myocardium. We also introduced $\lambda$-annealing schedules that control the relative weights of each of the four terms in our energy functional, so that the minimization process performs smoothly. Lastly, we validated the performance of STACS by comparing its segmentation results with the contours traced manually by an expert, assessing them quantitatively with the area and shape similarity measures. Both measures show very good agreement for all three contours across the 48 test images, i.e., across the 143 contours tested.

\section{APPENDIX I}

\section{EULER-LAGRANGE EQUATION FOR THE FUNCTIONAL $J(\phi)$}

In Section III, we need the Euler-Lagrange equation that minimizes the functional (29), which we restate here for convenience

$$
\begin{aligned}
J(\phi)= & \int_{\Omega} \lambda_{1} M_{1}(x, y) \mathcal{H}_{\epsilon}(\phi(x, y)) \\
& +\lambda_{1} M_{2}(x, y)\left[1-\mathcal{H}_{\epsilon}(\phi(x, y))\right] \\
& +P(x, y) \delta_{\epsilon}(\phi(x, y))\|\nabla \phi(x, y)\| d x d y
\end{aligned}
$$

where

$$
M_{k}(x, y)=\frac{1}{2} \ln \left(2 \pi \sigma_{k}^{2}\right)+\frac{\left(u(x, y)-m_{k}\right)^{2}}{2 \sigma_{k}^{2}}, \quad \text { for } k=1,2
$$

and

$$
P(x, y)=\lambda_{2} \Upsilon(x, y)+\lambda_{3} \mathcal{D}^{2}(x, y)+\lambda_{4}
$$

According to the Calculus of Variation [33], [21], the 2-D scalar function $\phi(x, y)$ that minimizes the functional (56) corresponds to the one that solves the PDE

$$
\frac{\partial E}{\partial \phi}-\frac{d}{d x}\left(\frac{\partial E}{\partial \phi_{x}}\right)-\frac{d}{d y}\left(\frac{\partial E}{\partial \phi_{y}}\right)=0
$$

where

$$
\begin{aligned}
E\left(\phi, \phi_{x}, \phi_{y}\right)=\lambda_{1} M_{1} \mathcal{H}_{\epsilon}(\phi)+\lambda_{1} M_{2}\left[1-\mathcal{H}_{\epsilon}(\phi)\right] \\
+P \delta_{\epsilon}(\phi) \sqrt{\phi_{x}^{2}+\phi_{y}^{2}} .
\end{aligned}
$$

For notation simplicity, we have omitted the independent variables $(x, y)$ of $\phi, \phi_{x}, \phi_{y}, P, M_{1}$, and $M_{2}$. We also note that

$$
\|\nabla \phi(x, y)\|=\sqrt{\phi_{x}^{2}+\phi_{y}^{2}} .
$$

Taking the partial derivative of $E$ in (60) with respect to $\phi, \phi_{x}$, and $\phi_{y}$, respectively, we have

$$
\begin{aligned}
\frac{\partial E}{\partial \phi} & =\lambda_{1}\left(M_{1}-M_{2}\right) \delta_{\epsilon}(\phi)+P \delta_{\epsilon}^{\prime}(\phi) \sqrt{\phi_{x}^{2}+\phi_{y}^{2}} \\
\frac{\partial E}{\partial \phi_{x}} & =P \delta_{\epsilon}(\phi) \frac{\phi_{x}}{\|\nabla \phi(x, y)\|} \\
\frac{\partial E}{\partial \phi_{y}} & =P \delta_{\epsilon}(\phi) \frac{\phi_{y}}{\|\nabla \phi(x, y)\|}
\end{aligned}
$$

where $\delta_{\epsilon}^{\prime}(\phi)$ is the derivative of $\delta_{\epsilon}$ with respect to $\phi$.

The derivative of (63) with respect to $x$ is

$$
\begin{aligned}
\frac{d}{d x}\left(\frac{\partial E}{\partial \phi_{x}}\right)=P_{x} \delta_{\epsilon}(\phi) \frac{\phi_{x}}{\|\nabla \phi\|} & +P \delta_{\epsilon}^{\prime}(\phi) \frac{\phi_{x}^{2}}{\|\nabla \phi\|} \\
& +P \delta_{\epsilon}(\phi) \frac{d}{d x}\left(\frac{\phi_{x}}{\|\nabla \phi\|}\right) .
\end{aligned}
$$

Similarly, the derivative of (64) with respect to $y$ is

$$
\begin{aligned}
\frac{d}{d y}\left(\frac{\partial E}{\partial \phi_{y}}\right)=P_{y} \delta_{\epsilon}(\phi) \frac{\phi_{y}}{\|\nabla \phi\|} & +P \delta_{\epsilon}^{\prime}(\phi) \frac{\phi_{y}^{2}}{\|\nabla \phi\|} \\
& +P \delta_{\epsilon}(\phi) \frac{d}{d y}\left(\frac{\phi_{y}}{\|\nabla \phi\|}\right) .
\end{aligned}
$$

Substituting (62), (65), and (66) back into the Euler-Lagrange (59) and utilizing the fact that

$$
\frac{\phi_{x}^{2}}{\|\nabla \phi\|}+\frac{\phi_{y}^{2}}{\|\nabla \phi\|}=\|\nabla \phi\|
$$

we have

$$
\left[\begin{array}{c}
\lambda_{1}\left(M_{1}-M_{2}\right) \\
-P_{x} \frac{\phi_{x}}{\|\nabla \phi\|}-P_{y} \frac{\phi_{y}}{\|\nabla \phi\|} \\
-P \frac{d}{d x}\left(\frac{\phi_{x}}{\|\nabla \phi\|}\right)-P \frac{d}{d y}\left(\frac{\phi_{y}}{\|\nabla \phi\|}\right)
\end{array}\right] \delta_{\epsilon}(\phi)=0 .
$$


We observe that

$$
P_{x} \frac{\phi_{x}}{\|\nabla \phi\|}+P_{y} \frac{\phi_{y}}{\|\nabla \phi\|}=\nabla P \cdot \frac{\nabla \phi}{\|\nabla \phi\|}
$$

where $\cdot$ represents a vector dot product, and

$$
\frac{d}{d x}\left(\frac{\phi_{x}}{\|\nabla \phi\|}\right)+\frac{d}{d y}\left(\frac{\phi_{y}}{\|\nabla \phi\|}\right)=\operatorname{div}\left(\frac{\nabla \phi}{\|\nabla \phi\|}\right) .
$$

Therefore, a compact form of (68) is

$$
\left[\lambda_{1}\left(M_{1}-M_{2}\right)-\nabla P \cdot \frac{\nabla \phi}{\|\nabla \phi\|}-P \operatorname{div}\left(\frac{\nabla \phi}{\|\nabla \phi\|}\right)\right] \delta_{\epsilon}(\phi)=0 .
$$

This is the Euler-Lagrange equation corresponding to the functional (56).

\section{REFERENCES}

[1] S. Yoshida, K. Takeuchi, P. J. del Nido, and C. Ho, "Cardiac function of transplanted rat hearts using a working heart model assessed by magnetic resonance imaging," The J. Heart Lung Transplant, vol. 18, pp. 1054-1064, 1999.

[2] R. J. van der Geest and J. H. Reiber, "Quantification in cardiac MRI," J. Magn. Reson. Imag., vol. 10, pp. 602-608, 1999.

[3] R. I. Pettigrew, J. N. Oshinski, G. Chatzimavroudis, and W. T. Dixon, "MRI techniques for cardiovascular imaging," J. Magn. Reson. Imag., vol. 10, pp. 590-601, 1999.

[4] M. Kass, A. Witkin, and D. Terzopoulos, "Snakes: Active contour models," Int. J. Comput. Vis., vol. 1, no. 4, pp. 321-331, 1988.

[5] L. D. Cohen, "On active contour models and balloons," CVGIP: Image Understanding, vol. 53, no. 2, pp. 211-218, Mar. 1991.

[6] R. Malladi, J. A. Sethian, and B. Vemuri, "Shape modeling with front propagation: A level set approach," IEEE Trans. Pattern Anal. Machine Intell., vol. 17, no. 2, pp. 158-175, Feb. 1995.

[7] C. Xu and J. L. Prince, "Snakes, shapes, and gradient vector flow," IEEE Trans. Med. Imag., vol. 7, no. 3, pp. 359-369, Mar. 1998.

[8] D. Mumford and J. Shah, "Optimal approximations by piecewise smooth functions and associated variational problems," Commun. Pure Appl. Math., vol. 12, no. 4, 1989.

[9] T. F. Chan and L. A. Vese, "Active contours without edges," IEEE Trans. Image Processing, vol. 10, no. 2, pp. 266-277, Feb. 2001

[10] A. Tsai, A. Yezzi, W. M. Wells, III, C. Tempany, D. Tucker, A. Fan, W. E. L. Grimson, and A. Willsky, "A shape-based approach to the segmentation of medical imagery using level sets," IEEE Trans. Med. Imag., vol. 22, no. 2, pp. 137-154, Feb. 2003.

[11] M. E. Leventon, W. E. L. Grimson, and O. Faugeras, "Statistical shape influence in geodesic active contours," in Proc. IEEE Conf. Computer Vision and Pattern Recognition (CVPR), vol. 1, Jun. 2000, pp. 316-323.

[12] N. Paragios, "A level set approach for shape-driven segmentation and tracking of the left ventricle," IEEE Trans. Med. Imag., vol. 22, no. 6, pp. 773-776, Jun. 2003.
[13] C. Xu and J. L. Prince, "Gradient vector flow: A new external force for snakes," in Proc. IEEE Conf. Computer Vision and Pattern Recognition (CVPR), 1997, pp. 66-71.

[14] P. Perona and J. Malik, "Scale-space and edge detection using anisotopic diffusion,” IEEE Trans. Pattern Anal. Machine Intell., vol. 12, no. 7, Jul. 1990.

[15] L. Alvarez, P. L. Lions, and J. M. Morel, "Image selective smoothing and edge detection by nonlinear diffusion II," SIAM J. Numerical Anal., vol. 29 , no. 3, 1992 .

[16] L. Alvarez, F. Guichard, P.-L. Lions, and J.-M. Morel, "Axioms and fundamental equations of image processing," Arch. Rational Mech., vol. 123, no. 3, pp. 199-257, 1993.

[17] L. Rudin, S. Osher, and C. Fatemi, "Nonlinear total variation based noise removal algorithm," Physica, vol. 60, no. D, pp. 259-268, 1992.

[18] Y.-L. You and M. Kaveh, "Fourth-order pairtial differential equations for noise removal," IEEE Trans. Image Processing, vol. 9, no. 10, pp. 1723-1730, Oct. 2000.

[19] R. Malladi and J. A. Sethian, "Image processing: Flows under min/max curvature and mean curvature," Graphical Models Image Processing, vol. 58, no. 2, pp. 127-141, Mar. 1996.

[20] A. W. Fitzgibbon, M. Pilu, and R. B. Fisher, "Direct least square fitting of ellipses," IEEE Trans. Pattern Anal. Machine Intell., vol. 21, no. 5, pp. 476-480, 1999.

[21] G. Sapiro, Geometric Partial Differential Equations and Image Analysis. Cambridge, U.K.: Cambridge Univ. Press, 2001.

[22] J. A. Sethian, Level Set Methods and Fast Marching Methods. Cambridge, U.K.: Cambridge Univ. Press, 1999.

[23] L. W. Johnson, R. Dean Riess, and J. T. Arnold, Introduction to Linear Algebra, 5th ed. Reading, MA: Addison Wesley, Jul. 2001.

[24] C. Pluempitiwiriyawej, J. M. F. Moura, Y.-J. L. Wu, and C. Ho, "Fast detection of the heart in cardiac MR image sequences,", 2003, submitted for publication.

[25] A. P. Zijdenbos, B. M. Dawant, R. A. Margolin, and C. Palmer, "Morphometric anaysis of white matter lesions in MR images: Method and validation," IEEE Trans. Med. Imag., vol. 13, no. 4, Dec. 1994.

[26] M. Stella Atkins and B. T. Mackiewich, "Fully automatic segmentation of the brain in MRI," IEEE Trans. Med. Imag., vol. 17, no. 1, pp. 98-107, Feb. 1998.

[27] H. G. Barrow, J. M. Tenenbaum, R. C. Bolles, and H. C. Wolf, "Parametric correspondence and chamfer matching: Two techniques for image matching," in Proc. 5th Annu. Int. Joint Conf. Artificial Intelligence, Aug. 1977, pp. 659-663.

[28] Handbook of Medical Imaging: Processing and Analysis, I. N. Bankman, Ed., Academic, New York, 2000, pp. 515-527.

[29] J.-C. Cheng, "Capture and representation of human walking in live monocular video," Ph.D. thesis, Carnegie Mellon Univ., Pittsburgh, PA, Nov. 1998.

[30] J.-C. Cheng and J. M. F. Moura, "Automatic recognition of human walking in monocular image sequences," in J. VLSI Signal Porcessing, vol. 20, 1998, pp. 107-120.

[31] _ "Capture and representation of human motion in live video sequences," IEEE Trans. Multimedia, vol. 1, no. 2, pp. 144-156, Jun. 1999.

[32] O. Cuisenaire and B. Macq, "Fast and exact signed Euclidean distance transformation with linear complexity," in Proc. IEEE Int. Conf. Acous tics, Speech, and Signal Processing (ICASSP'99), vol. 6, Mar. 1999, pp. 3293-3296.

[33] L. E. Elsgolc, Calculus of Variations. Reading, MA: Addison-Wesley, 1962. 